Int. J. of The Soc. of Mat. Eng. for Resources Vol. 7 No. 1 166 177 (1999)

\title{
Original
}

\section{Three Dimensional Simulation for Extension of Interlaminar Delamination in Laminated Composite}

\author{
by \\ Yotsugi ShIBUYA $^{\dagger}$, Li Zhen $\mathrm{Sun}^{\dagger}$ and Tadashi OHyoshl ${ }^{\dagger}$
}

\begin{abstract}
An effective numerical algorithm is developed to simulate extension of the interlaminar delamination in a laminated composite based on the three dimensional finite element method. As use of the conjugate gradient or ICCG method to solve a system of linear equations minimizes occupation of memory space in computation, matrix of linear equations is made to be symmetric for contact problems on the delaminated interface of the composite. The extension of the interlaminar delamination is simulated in a cross-ply laminated composite with four layers under concentrated load on the center of the composite. Distribution of deflection on the laminated composite with the delamination is also shown graphically.
\end{abstract}

Key Words : Laminated Composite, Interlaminar Delamination, Contact Problem, Finite Element Method, Three Dimensional Analysis.

\section{Introduction}

Laminated fiber-reinforced composite materials have been used in aircraft and space vehicles, where high strength-to-weight and stiffness-to-weight ratios are required. As the composite materials are damaged due to impact, the compressive strength of composite materials is reduced remarkably. This phenomenon is a problem in laminated composites, and called Compression After Impact (CAI). Since an aircraft collides with birds or volcanic ash in the course of flight, a critical problem may arise in the practical application of composite materials. In fact, the ever-increasing use of composite materials in technological areas, where both precision and reliability play important roles, has required a better understanding of the behavior of the composite materials in a severe structural environment. Impact-induced delamination is one of frequent damage modes in laminated composites. It is necessary to study the damage of laminated fiber composite material after impact. Along with the explosive development of computational devices, it has become possible to simulate interior delamination by numerical methods.

Extensive studies, both experimental and analytical, have been conducted to determine the relation-

Received August 5, 1998

† Department of Mechanical Engineering, Faculty of Engineering and Resource Science, Akita University, 1-1, Tegatagakuencho, Akita, 010-8502 JAPAN. 
ship between the level of impact and the extent of delamination. Many researchers, for example, Joshi and Sun $^{1}$, Mukherjee et al. ${ }^{2}$, Liu and Chang ${ }^{3}$, Collombet et al. ${ }^{4}$ have studied the damage of composites after the impact loading. Wang and $\mathrm{Khanh}^{5}$ report the impact tests to predict the delamination size for dynamic fracture. Lu and Liu ${ }^{6}$ develop a 3-D finite element program to calculate the strain energy release rates at the delamination front and they suggest the mode II of fracture is the most important damage mode for central delamination. Studies of the finite element method have been reported to be used to simulate the damaged composites on computers. Robbins and Reddy ${ }^{7}$ report a useful finite element method for thick composites using a layerwise laminated theory. In order to analyze free edge stresses and delamination of composite laminates using a three-dimensional finite element, the preconditioned conjugate gradient method is used by Yang and $\mathrm{He}^{8}$ to solve the large system of linear equations.

In this paper, an effective numerical method is presented to simulate extension of the interfacial delamination in a laminated composite based on the three dimensional finite element method. An iterative algorithm and duplicate nodes assigned at the interface are used to represent the newly extended delamination. As use of the conjugate gradient or ICCG method to solve a system of linear equations minimizes occupation of memory space in computation, we attempt to make the matrix symmetric in the linear equations for contact problem on the delaminated interface of the composite. The extension of interlaminar delamination is simulated in a cross-play laminated composite with four layers. This composite is subjected to a point load on the center. In the numerical calculation, a cross-ply laminated composite of carbon/epoxy with four layers is treated. Extension of delamination and distribution of deflection in the composite are shown.

\section{Statement of the Problem}

A model of laminated composite materials is established in order to simulate its behavior of delamination under out-of-plane loading. We attempt to predict the delaminated area using a numerical method. Cartesian coordinate system $\left(x_{1}, x_{2}, x_{3}\right)$ is supposed and a laminated composite plate is placed in the $x_{1}-x_{2}$ plane as shown in Fig. 1 (a). The plate consists of $n$ layers with different directions of fibers, each layer being a unidirectional fiber-reinforced composite. The lengths of $x_{1}$ and $x_{2}$ directions are $2 L_{1}$ and $2 L_{2}$, respectively, and the height of the plate is $H$. Here, the thickness of a layer is $h(=H / n)$. The fiber angle of the $i$-th layer is $\theta_{i}$ against the $x_{1}$ axis. The layers of the plate are

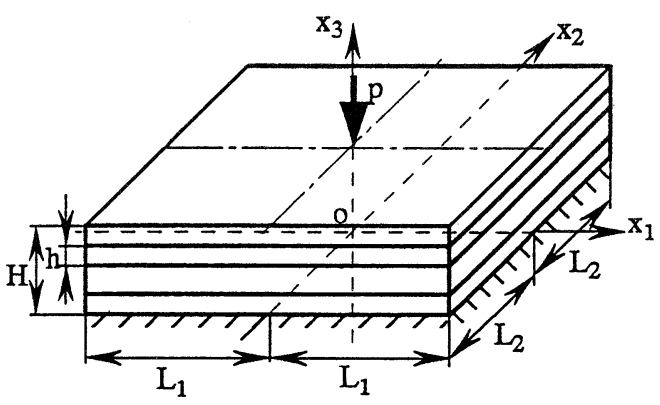

(a) Geometry of a laminated composite

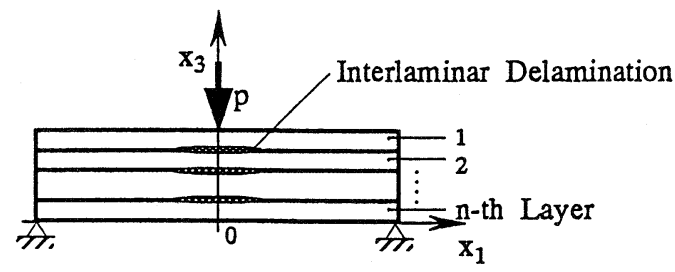

(b) Cross section at $x_{2}=0$

Fig. 1 Geometry of a laminated fiber-reinforced composite subjected to a concentrated load p. 
built up with different direction of fiber for each layer, and interlaminar delamination is considered at the interface of the layers due to loading as shown in Fig. 1 (b).

For the composite, the relation between strain and displacement is written as:

$$
\varepsilon_{i j}=\frac{1}{2}\left(\frac{\partial u_{i}}{\partial x_{j}}+\frac{\partial u_{j}}{\partial x_{i}}\right),(i, j=1,2,3)
$$

where $\varepsilon_{i j}$ are strain components, and $u_{i}$ are displacement components. When a layer is assumed to be transversely isotropic material, the relation between stress and strain is given for $\theta=0^{\circ}$ as follows :

$$
\{\sigma\}=[C]\{\varepsilon\}
$$

where $\{\sigma\}=\left\{\sigma_{11} \sigma_{22} \sigma_{33} \sigma_{12} \sigma_{23} \sigma_{13}\right\}^{T}$ is the stress vector, [C] is the stiffness matrix and $\{\varepsilon\}=\left\{\varepsilon_{11}\right.$ $\left.\begin{array}{llllll}\varepsilon_{22} & \varepsilon_{33} & \varepsilon_{12} & \varepsilon_{23} & \varepsilon_{13}\end{array}\right\}^{T}$ is the strain vector. Here,

$$
[C]=\left[\begin{array}{cccccc}
C_{11} & C_{12} & C_{12} & 0 & 0 & 0 \\
C_{12} & C_{22} & C_{23} & 0 & 0 & 0 \\
C_{12} & C_{23} & C_{22} & 0 & 0 & 0 \\
0 & 0 & 0 & 2 C_{66} & 0 & 0 \\
0 & 0 & 0 & 0 & C_{22}-C_{23} & 0 \\
0 & 0 & 0 & 0 & 0 & 2 C_{66}
\end{array}\right]
$$

$C_{i j}$ are components of elastic moduli. Relations among $C_{i j}$ and engineering moduli are as follows ${ }^{9}$ :

$$
\begin{aligned}
& C_{11}=E_{11}+4 v_{12}^{2} K_{23} \\
& C_{12}=4 v_{12} K_{23} \\
& C_{22}=\mu_{23}+K_{23} \\
& C_{23}=-\mu_{23}+K_{23} \\
& C_{66}=\mu_{12}
\end{aligned}
$$

and

$$
K_{23}=\frac{\left(1+v_{23}\right) E_{11} \mu_{23}}{\left(1-v_{23}\right) E_{11}-4\left(1+v_{23}\right) v_{12}^{2} \mu_{23}}
$$

where $E_{11}$ is Young's modulus, $v_{12}$ and $v_{23}$ are Poisson's ratios, $\mu_{12}$ and $\mu_{23}$ are shear moduli.

For $i$-th layer with $\theta_{i}$, the relation between stress and strain is

$$
\{\sigma\}=\left[Q_{i}\right]^{-1}[C]\left[Q_{i}\right]\{\varepsilon\}
$$

where $\left[Q_{i}\right]$ is the transformation matrix as follows :

$$
\left[Q_{i}\right]=\left[\begin{array}{cccccc}
\cos ^{2} \theta_{i} & \sin ^{2} \theta_{i} & 0 & 2 \cos \theta_{i} \sin \theta_{i} & 0 & 0 \\
\sin ^{2} \theta_{i} & \cos ^{2} \theta_{i} & 0 & -2 \sin \theta_{i} \cos \theta_{i} & 0 & 0 \\
0 & 0 & 1 & 0 & 0 & 0 \\
-\cos \theta_{i} \sin \theta_{i} & \sin \theta_{i} \cos \theta_{i} & 0 & \cos ^{2} \theta_{i}-\sin ^{2} \theta_{i} & 0 & 0 \\
0 & 0 & 0 & 0 & \cos \theta_{i} & -\sin \theta_{i} \\
0 & 0 & 0 & 0 & \sin \theta_{i} & \cos \theta_{i}
\end{array}\right]
$$


Next, it is assumed that the friction on the surface of delamination crack is free and the crack is always closed. Boundary conditions at the interface without delamination are written as:

$$
\left.\begin{array}{rl}
u_{j}^{i} & =u_{j}^{i+1},(j=1,2,3) \\
\sigma_{13}^{i} & =\sigma_{13}^{i+1} \\
\sigma_{23}^{i} & =\sigma_{23}^{i+1} \\
\sigma_{33}^{i} & =\sigma_{13}^{i+1}
\end{array}\right\}
$$

where superscripts $i$ and $(i+1)$ indicate $i$-th and $(i+1)$-th layers at the interface, respectively. The boundary conditions on the surface of the delamination cracks are given as:

$$
\left.\begin{array}{rl}
u_{3}^{i} & =u_{3}^{i+1} \\
\sigma_{23} & =0 \\
\sigma_{13} & =0 \\
\sigma_{33}^{i} & =\sigma_{33}^{i+1}
\end{array}\right\}
$$

The boundary conditions for supporting of the panel at edges, i. e., $\left(\mathrm{x}_{1}= \pm \mathrm{L}_{1},-\mathrm{L}_{2} \leq \mathrm{x}_{2} \leq \mathrm{L}_{2}, \mathrm{x}_{3}=\right.$ $0)$ and $\left(-\mathrm{L}_{1} \leq \mathrm{x}_{1} \leq \mathrm{L}_{1}, \mathrm{x}_{2}= \pm \mathrm{L}_{2}, \mathrm{x}_{3}=0\right)$, are assumed as follows :

$$
u_{i}=0,(j=1,2,3)
$$

The applied loading $p\left(x_{1}, x_{2}, x_{3}\right)$ is exerted on the center of the upper surface of the plate. Here,

$$
p\left(x_{1}, x_{2}, x_{3}\right)=-P \delta\left(x_{1}\right) \delta\left(x_{2}\right) \delta\left(x_{3}-H\right)
$$

where $\delta()$ is Dirac's delta function. $P$ is the intensity of the load.

\section{Finite Element Analysis for Interlaminar Delamination}

\section{1 Finite Element Modeling}

The interlaminar delamination of the composite is analyzed by three dimensional finite element method. A laminated composite with four layers is treated in the numerical calculation. In view of symmetrical structure of the problem, a quarter of the plate is divided into finite element mesh with 8-node solid elements as shown in Fig. 2. Small elements are placed in the center area and the relatively large elements are arranged outside the region.

Consider the upper and lower layers of the interface in the composite as shown in Fig. 3 (a). Where $p_{k}$ and $q_{k}$ are the nodal points of the upper and lower layers at the interface. For bonding layers together, the duplicate nodes with the numbers $p_{k}$ and $q_{k}$ are made to represent separable interface as shown in Fig. 3 (b). When the delaminated region extends up to the point $p_{k}, q_{k}$ as shown in Fig. 3 (c), points $p_{k-1}$ and $q_{k-1}, \ldots$ will be slidden due to delamination. The delamination at the interface is forced to proceed by separating the duplicate node at the front of delaminated region $p_{k}$ and $q_{k}$ in the Fig. 3 (c).

\section{2 Numerical Procedure on the Interface}

For the interface between layers, boundary conditions are given in Eqs. (8) and (9). The boundary conditions can be rewritten using nodal forces and displacements for finite element analysis at the interface between the $i$-th and $(i+1)$-th layers at the nodal points $p_{k}$ and $q_{k}$. 


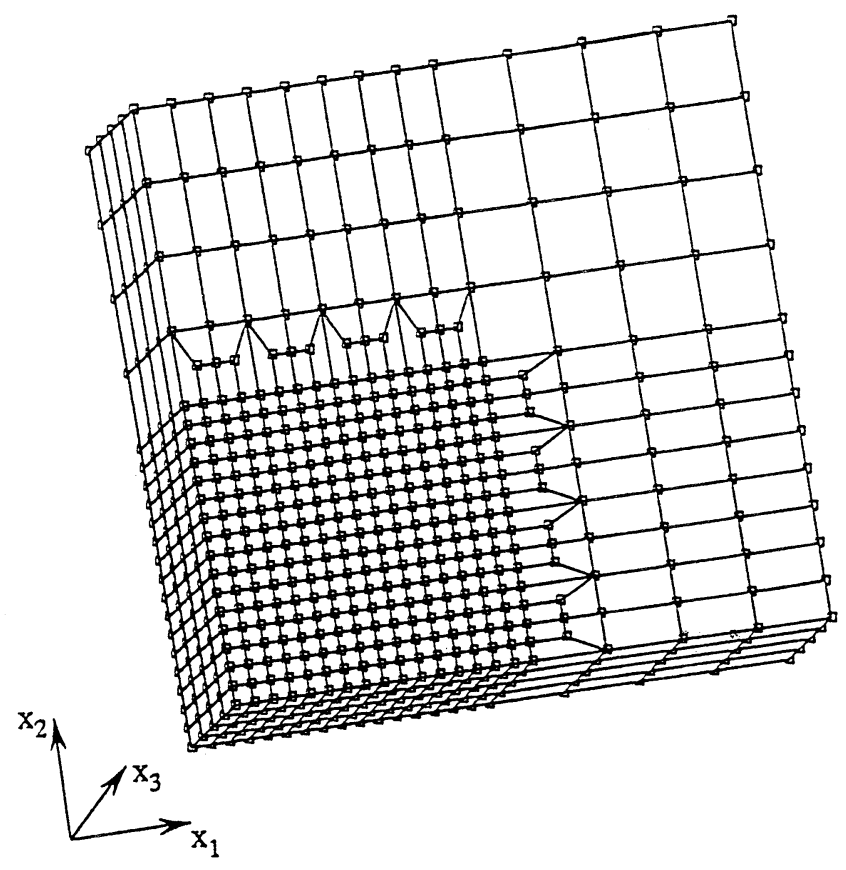

Fig. 2 Finite element mesh for a quarter model.

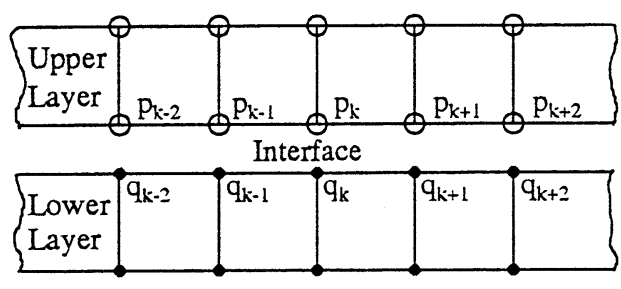

(a) Mesh for two layers

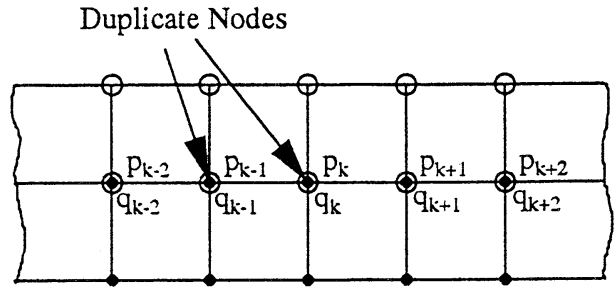

(b) Before delamination

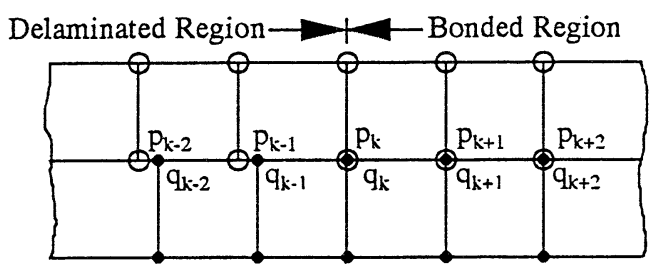

(c) After delamination

Fig. 3 Duplicate nodes for analysis of delamination at the interface. 
(a) At interface without delamination

$$
\left.\begin{array}{l}
u_{j}^{i}\left(p_{k}\right)=u_{j}^{i+1}\left(q_{k}\right) \\
f_{j}^{i}\left(p_{k}\right)=-f_{j}^{i+1}\left(q_{k}\right)
\end{array}\right\}(j=1,2,3)
$$

(b) At interface with delamination

$$
\left.\begin{array}{l}
u_{3}^{i}\left(p_{k}\right)=u_{3}^{i+1}\left(q_{k}\right) \\
f_{3}^{i}\left(p_{k}\right)=f_{3}^{i+1}\left(q_{k}\right) \\
f_{j}^{i}\left(p_{k}\right)=-f_{j}^{i+1}\left(q_{k}\right),(j=1,2)
\end{array}\right\}
$$

The total stiffness equation in the finite element method can be written as follows :

$$
[K]\{u\}=\{f\}
$$

where $[K]$ represents the total stiffness matrix, and $\{u\}$ and $\{f\}$ represent nodal displacement and nodal force vectors, respectively. The total stiffness matrix is usually symmetrical. If boundary conditions (12) and (13) corresponding to a contact problem are imposed into Eq. (14) symmetrically, the storage space for the coefficient matrix will be reduced to half of the whole coefficient matrix and the conjugate gradient method is applied to solve the linear equations easily. This makes occupation of memory space in the computation minimum.

In this study, a technique of computation is developed in order to create symmetric matrix of coefficients which satisfies boundary conditions $u_{j}^{i}\left(p_{k}\right)=u_{j}^{i+1}\left(q_{k}\right)$ and $f_{j}^{i}\left(p_{k}\right)=-f_{j}^{i+1}\left(q_{k}\right)$ in the Eq. (12). For the symmetric total stiffness matrix in Fig. 4 , coefficients of $n_{1}$-th and $n_{2}$-th columns correspond to the nodal displacements $u_{j}^{i}\left(p_{k}\right)$ and $u_{j}^{i+1}\left(q_{k}\right)$, and coefficients of $n_{1}$-th and $n_{2}$-th rows correspond to the nodal forces $f_{j}^{i}\left(p_{k}\right)$ and $f_{j}^{i+1}\left(q_{k}\right)$, respectively.

The procedure to impose the boundary conditions into the matrix $[K]$ is summarized as follows :

(1) From $u_{j}^{i}\left(p_{k}\right)=u_{j}^{i+1}\left(q_{k}\right)$, the $n_{2}$-th column is added to $n_{1}$-th column and $n_{2}$-th column is set to zero as :

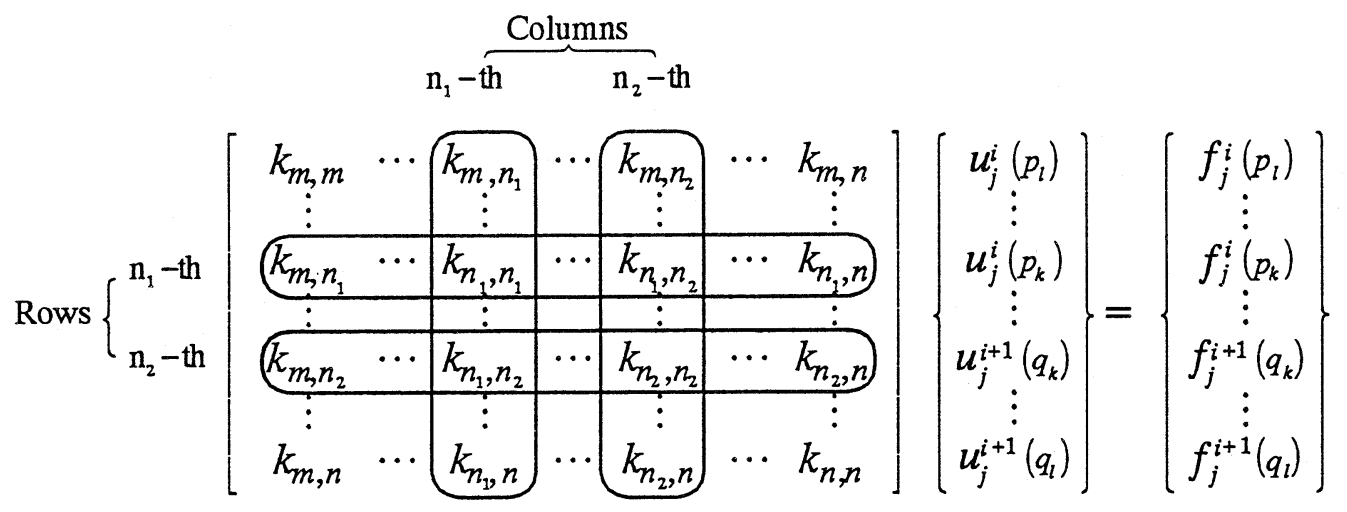

Fig. 4 Total stiffness equation of the finite element method. 


$$
\left[\begin{array}{ccccccc}
k_{m, m} & \cdots & k_{m, n_{1}}+k_{m, n_{2}} & \cdots & 0 & \cdots & k_{m, m} \\
\vdots & & \vdots & & \vdots & & \vdots \\
k_{m, n_{1}} & \cdots & k_{n_{1}, n_{1}}+k_{n_{1}, n_{2}} & \cdots & 0 & \cdots & k_{n_{1}, n} \\
\vdots & & \vdots & & \vdots & & \vdots \\
k_{m, n_{2}} & \cdots & k_{n_{1}, n_{2}}+k_{n_{2}, n_{2}} & \cdots & 0 & \cdots & k_{n_{2}, n} \\
\vdots & & \vdots & & \vdots & & \vdots \\
k_{m, n} & \cdots & k_{n_{1}, n}+k_{n_{2}, n} & \cdots & 0 & \cdots & k_{n, n}
\end{array}\right]\left\{\begin{array}{c}
u_{j}^{i}\left(p_{l}\right) \\
\vdots \\
u_{j}^{i}\left(p_{k}\right) \\
\vdots \\
u_{j}^{i+1}\left(q_{k}\right) \\
\vdots \\
u_{j}^{i+1}\left(q_{l}\right)
\end{array}\right\}=\left\{\begin{array}{c}
f_{j}^{i}\left(p_{l}\right) \\
\vdots \\
f_{j}^{i}\left(p_{k}\right) \\
\vdots \\
f_{j}^{i+1}\left(q_{k}\right) \\
\vdots \\
f_{j}^{i+1}\left(q_{l}\right)
\end{array}\right\}
$$

(2) For $f_{j}^{i}\left(p_{k}\right)=-f_{j}^{i+1}\left(q_{k}\right)$, the $n_{2}$-th row is added to $n_{1}$-th row and $n_{2}$-th row is set to zero, and the matrix $[K]$ is

$\left[\begin{array}{ccccccc}k_{m, m} & \cdots & k_{m, n_{1}}+k_{m, n_{2}} & \cdots & 0 & \cdots & k_{m, m} \\ \vdots & & \vdots & & \vdots & & \vdots \\ k_{m, n_{1}}+k_{m, n_{2}} & \cdots & k_{n_{1}, n_{1}}+k_{n_{1}, n_{2}}+k_{n_{1}, n_{2}}+k_{n_{2}, n_{2}} & \cdots & 0 & \cdots & k_{n_{1}, n_{n}}+k_{n_{2}, n} \\ \vdots & & \vdots & & \vdots & & \vdots \\ 0 & \cdots & 0 & \cdots & 0 & \cdots & 0 \\ \vdots & & \vdots & & \vdots & & \vdots \\ k_{m, n} & \cdots & k_{n_{1}, n}+k_{n_{2}, n} & \cdots & 0 & \cdots & k_{n, n}\end{array}\right]$

(3) For $u_{j}^{i}\left(p_{k}\right)=u_{j}^{i+1}\left(q_{k}\right)$, the $n_{1}$-th and $n_{2}$-th components of the $n_{2}$-th row is set to -1 and 1 , respectively, as follows:

$$
\left[\begin{array}{ccccccc}
k_{m, m} & \cdots & k_{m, n_{1}}+k_{m, n_{2}} & \cdots & 0 & \cdots & k_{m, m} \\
\vdots & & \vdots & & \vdots & & \vdots \\
k_{m, n_{1}}+k_{m, n_{2}} & \cdots & k_{n_{1}, n_{1}}+k_{n_{1}, n_{2}}+k_{n_{1}, n_{2}}+k_{n_{2}, n_{2}} & \cdots & 0 & \cdots & k_{n_{1}, n}+k_{n_{2}, n} \\
\vdots & & \vdots & & \vdots & & \vdots \\
0 & \cdots & -1 & \cdots & 1 & \cdots & 0 \\
\vdots & & \vdots & & \vdots & & \vdots \\
k_{m, n} & \cdots & k_{n_{1}, n}+k_{n_{2}, n} & \cdots & 0 & \cdots & k_{n, n}
\end{array}\right]
$$

(4) In order to make the matrix symmetric, the $n_{1}$-th and $n_{2}$-th components of the $n_{1}$-th row are added to 1 and -1 , respectively, as follows :

$$
\left[\begin{array}{ccccccc}
k_{m, m} & \cdots & k_{m, n_{1}}+k_{m, n_{2}} & \cdots & 0 & \cdots & k_{m, m} \\
\vdots & & \vdots & & \vdots & & \vdots \\
k_{m, n_{1}}+k_{m, n_{2}} & \cdots & k_{n_{1}, n_{1}}+k_{n_{1}, n_{2}}+k_{n_{1}, n_{2}}+k_{n_{2}, n_{2}}+1 & \cdots & -1 & \cdots & k_{n_{1}, n_{n}}+k_{n_{2}, n} \\
\vdots & & \vdots & & \vdots & & \vdots \\
0 & \cdots & -1 & \cdots & 1 & \cdots & 0 \\
\vdots & & \vdots & & \vdots & & \vdots \\
k_{m, n} & \cdots & k_{n_{1}, n}+k_{n_{2}, n} & \cdots & 0 & \cdots & k_{n, n}
\end{array}\right]
$$

This technique is not only for keeping the symmetry of the original matrix $[K]$, but also for satisfying the boundary conditions at the interface. 


\section{Extension of Delamination}

\section{1 Energy Release Rate}

An interlaminar delamination tends to grow when an applied stress reaches a critical value. The energy release rate $G$ could be computed based on the change of potential energy or the change of compliance for two crack lengths by the following basic equation at the crack tip :

$$
G=d U / d a
$$

where $d U$ is the increment of surface energy, and $d a$ is the area of an infinitesimal extension of the crack.

The energy release rate based on the finite element method is presented by the crack closure method where nodal forces and displacements are employed. The energy release rate can be calculated for shear mode by the following equation :

$$
G=\frac{1}{2 \Delta a}\left(\Delta u_{1} f_{1}+\Delta u_{2} f_{2}\right)
$$

where $\Delta a$ is the area of the extension of the crack, $f_{1}$ and $f_{2}$ are the nodal forces of the $x_{1}$ and $x_{2}$ directions at the interface, respectively. $\Delta u_{1}$ and $\Delta u_{2}$ are gaps of nodal displacements of the $x_{1}$ and $x_{2}$ directions at the interface, respectively .

\section{2 Algorithm for Extension of the Delamination}

Although the extension of delamination may obey the energy release rate, the algorithm for extension with a concept of the energy release rate needs a large amount of iterative process in the simulation. In this study, it is assumed that newly extended delamination occurs at a location, where a value associated with shear nodal force is maximum among nodes. These nodes are along front of

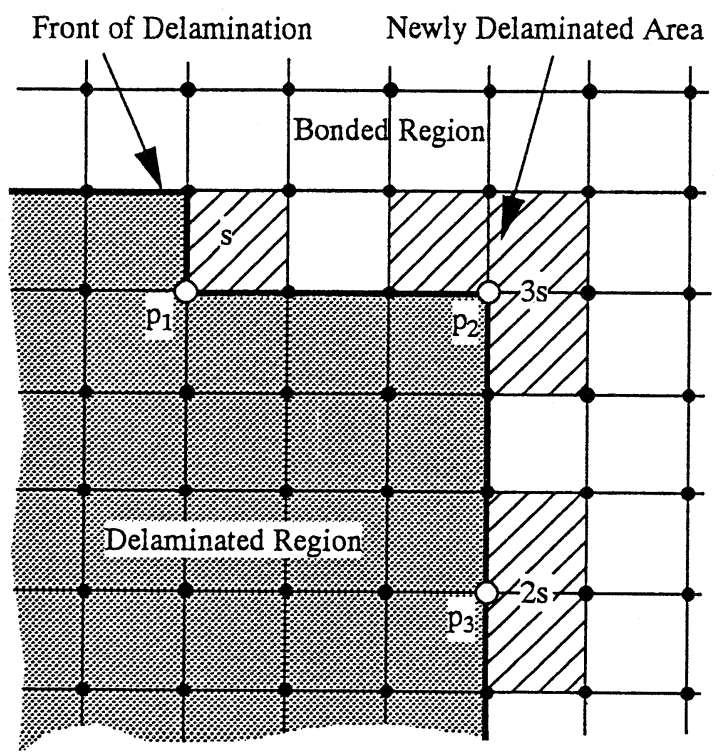

Fig. 5 Illustration of extension of delamination. 
the delamination in Fig. 5. The value $F$ is expressed as

$$
\mathrm{F}=\sqrt{\left[f_{1}^{i}\left(\boldsymbol{p}_{\boldsymbol{k}}\right)\right]^{2}+\left[f_{2}^{i}\left(\boldsymbol{p}_{\boldsymbol{k}}\right)\right]^{2}} / A
$$

where $A$ is the area of newly extended delamination due to separation of a duplicate node. The area varies with geometry of delamination. For example, $A$ is for separation at point $p_{1}, 3 s$ at $p_{2}$ and $2 s$ at point $p_{3}$ as shown in Fig. 5, respectively. Here, $s$ is the area of a square region of an element. The simulation for extension of delamination is made iteratively by determining the separated node from Eq. (21).

\section{Numerical Results and Discussion}

Extension of interlaminar delamination in the cross-ply laminated composite with four layers is simulated on the basis of the aforementioned technique. The simulation is carried out iteratively due to the extension of the interlaminar delamination. In one step of iterations on the simulation, separation at a duplicable node of the interface is assumed as a new small extension of the delamination.

The length, the width and the height of the plate used in the numerical calculation are conferred to be $L_{1}=50 \mathrm{~mm}, L_{2}=50 \mathrm{~mm}, H=0.8 \mathrm{~mm}$. The plate consists of four layers, the thickness of one layer is $h=0.2 \mathrm{~mm}$. The angles of fiber for layers are $\theta_{1}=\theta_{3}=0^{\circ}$ and $\theta_{2}=\theta_{4}=90^{\circ}$, as a cross-ply laminated composite of a unidirectional carbon/epoxy composite. The lamina of composite is transversely isotropic and the following properties are used: $E_{11}=134 \mathrm{GPa}, \mu_{12}=5.52 \mathrm{GPa}, \mu_{23}=3.43 \mathrm{GPa}, v_{12}=$ $0.3, v_{12}=0.49$.

Figure 6 shows patterns of the delamination at the interface between the second and third layer of the composite, i.e., the central interface of the composite. The area of the delamination at the interface is indicated by hatched and dotted regions. In the figure, (a), (b), (c) and (d) are in cases

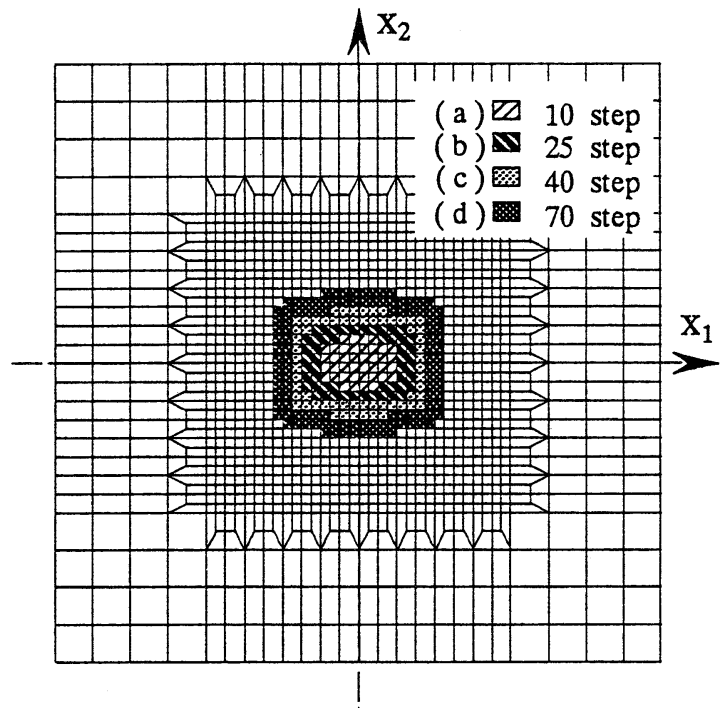

Fig. 6 Patterns of delamination at the interface between the second layer and the third layer of the laminated composite. 


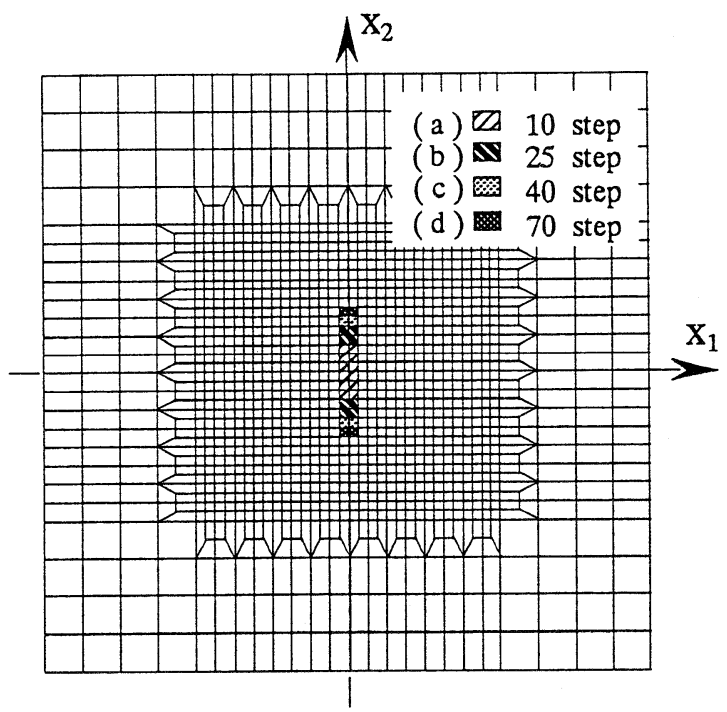

Fig. 7 Patterns of delamination at the interface between the third layer and the fourth layer of the laminated composite.

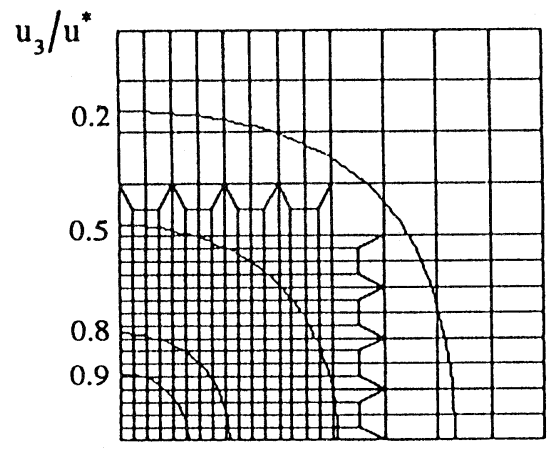

(a) $S_{d}=0.0 \mathrm{~mm}^{2}$ (0 step)

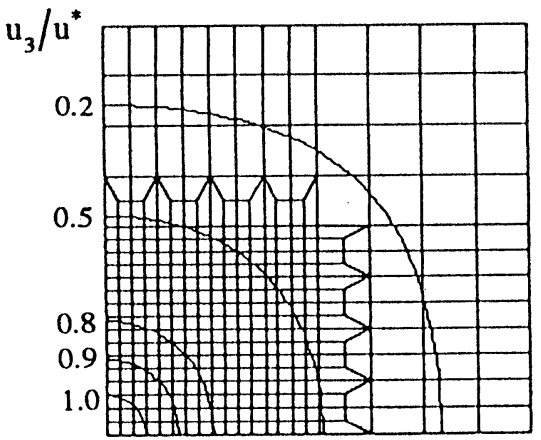

(c) $S_{d}=410.0 \mathrm{~mm}^{2}$ (40 step)

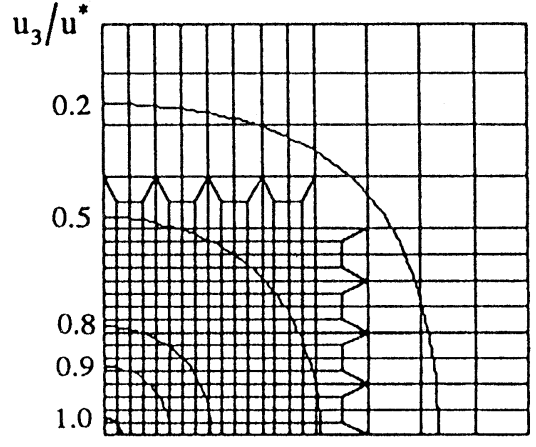

(b) $\mathrm{S}_{\mathrm{d}}=107.2 \mathrm{~mm}^{2}$ (10 step)

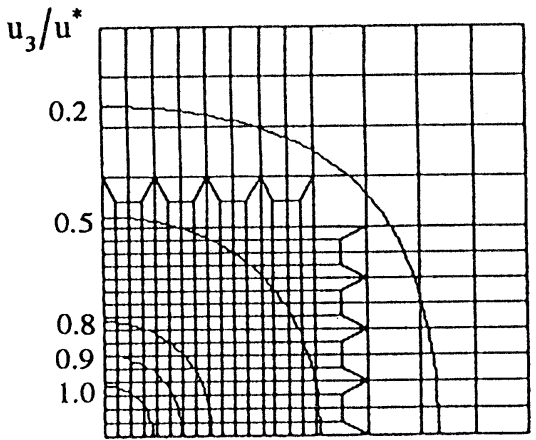

(d) $\mathrm{S}_{\mathrm{d}}=693.2 \mathrm{~mm}^{2}$ (70 step)

Fig. 8 Distributions of deflection $\mathrm{u}_{3} / u^{*}$ in the laminated composite. 
of up to 10 step, 25 step, 40 step and 70 step on the iteration, respectively. Total area $S_{d}$ of the interlaminar delamination in the composite is also calculated. The areas which correspond to the 10 step, 25 step, 40 step, and 70 step are $S_{d}=107.2 \mathrm{~mm}^{2}, 253.9 \mathrm{~mm}^{2}, 410.1 \mathrm{~mm}^{2}$ and $639.4 \mathrm{~mm}^{2}$, respectively. The pattern of the delamination is similar to rectangular shape or ellipse shape. The behavior of the delamination tends to extend more in the $x_{1}$ direction, which corresponds to the fiber direction of the third layer.

Similar to Fig. 6, pattern of the delamination at the interface between the third and fourth layers, i.e., lower interface of the composite, is shown in Fig. 7. The principal direction of the extension of the delamination is the $x_{2}$ direction which corresponds to the fiber direction of the fourth layer. As a tendency from Fig. 6 and Fig. 7, the direction of the extension depends on the fiber direction of the lower layer at the interface. A large area of the delamination takes place at the central interface of the composite and the area of the delamination at the lower interface is small. Moreover, for the upper interface, i.e., the interface between the first and second layers, the extension of the delamination does not take place in the range of this simulation.

In a real laminated composite, a matrix-rich interphase exists between layers. However, the interphase layer can be considered as a part of the upper layer, because the longitudinal Young's modulus of lower layer is very large in comparison with the transverse Young's moduli of the upper layer. Then the interface of the lower layer and interphase layer becomes important to estimate the delamination. The numerical simulation certifies this fact to the tendency to delaminate along the fiber direction.

Figure 8 shows distribution of deflection $\mathrm{u}_{3}$ of the composite at $x_{3}=H$ normalized by deflection $u^{*}$ of a delamination-free composite at the loading point. Case of delamination free is shown in Fig. 8 (a) as a reference, i.e., $S_{d}=0$. Deflections far from the loading points are almost the same in the cases, for example, $\mathrm{u}_{3} / u^{*}=0.2$ and 0.5 . However, the deflection increases with the increasing of the area of the delamination near the loading point. Contours for $u_{3} / u^{*}=1.0$ and 0.9 tend to extend with increase of $S_{d}$.

This model contains a total of 1,472 solid elements and 2,773 nodal points with 8,319 degrees of freedom. The occupation of memory to save coefficients of linear equations in the actual calculation is 457,488 words for the conjugate gradient method. The storage size is about $0.7 \%$ of that needed for full matrix.

\section{Conclusions}

Extension of interlaminar delamination in the laminated composite is studied in this paper. An effective numerical algorithm is developed on the basis of three dimensional finite element method. Boundary conditions at the interface for delamination, which correspond to a contact problem, are imposed into coefficient matrix of linear equations symmetrically, and the conjugate gradient method is applied to solve the linear equations easily. This contributes to minimize occupation of memory space in computation. The extensions of the interlaminar delamination for delamination is discussed. It suggests in the simulation that direction of major extension of the delamination corresponds to the fiber direction of lower layer at the interface. 


\section{References}

1. S. P. Joshi and C. T. Sun, 'Impact Induced Fracture in a Laminated Composite', J. Compos. Mater., 19, 51-66 (1985).

2. Y. X. Mukherjee, S. N. Gulrajani, S. Mukherjee and A. N. Netravali, 'A Numerical and Experimental Study of Delaminated Layered Composites', J. Compos. Mater., 28, 837-870 (1994).

3. S. Liu and F. K. Chang, 'Matrix Cracking Effect on Delamination Growth in Composite Laminates Induced by a Spherical Indenter', J. Compos. Mater., 28, 940-977 (1994).

4. F. Collombet, J. Bonini and J. L. Lataillade, 'A Three-Dimensional Modeling of Low Velocity Impact Damage in Composite Laminates', Int. J. Numer. Methods Eng., 39, 1491-1516 (1996).

5. H. Wang and T. U. Khanh, 'Fracture Mechanics and Mechanisms of Impact-Induced Delamination in Laminated Composite', J. Compos. Mater., 29, 156-178 (1995).

6. L. Q. Lu and D. Liu, 'Finite Element Analysis of Strain Energy Release Rate at Delamination Front', J. Reinforced Plastics and Compos., 10, 279-292 (1991).

7. D. H. Jr. Robbins and J. N. Reddy, 'Modeling of Thick Composites Using a Layerwise Laminate Theory', Int. J. Numer. Methods Eng., 36, 655-677 (1993).

8. H. T. Y. Yang and C.C. He, 'Three-Dimensional Finite Element Analysis of Free Edge Stresses and Delamination of Composite Laminates', J. Compos. Mater., 28, 1394-1412 (1994).

9. R. M. Christensen, Mechanics of Composite Materials, Krieger Publishing, Florida, 1991.

10. K. Kageyama, M. Kikuchi and N. Yanagisawa, 'Stabilized End Notched Flexure Test : Characterization of Mode II Interlaminar Crack Growth', Compos. Mater. : Fatigue and Fracture (Third Volume), ASTM STP 1110, 210-225 (1991). 